\title{
Electrocaloric characterization of a poly(vinylidene fluoride- trifluoroethylene-chlorofluoroethylene) terpolymer by infrared imaging
}

\author{
Dongzhi Guo, ${ }^{1, a)}$ Jinsheng Gao, ${ }^{2, a)}$ Ying-Ju Yu, ${ }^{1}$ Suresh Santhanam, ${ }^{2}$ Gary K. Fedder, ${ }^{2}$ \\ Alan J. H. McGaughey, ${ }^{1}$ and S. C. Yao ${ }^{1, b)}$ \\ ${ }^{1}$ Department of Mechanical Engineering, Carnegie Mellon University, Pittsburgh, Pennsylvania 15213-3890, \\ USA \\ ${ }^{2}$ Department of Electrical and Computer Engineering, Carnegie Mellon University, Pittsburgh, \\ Pennsylvania 15213-3890, USA
}

(Received 16 June 2014; accepted 8 July 2014; published online 21 July 2014)

\begin{abstract}
The electrocaloric effect in thin films of a poly(vinylidene fluoride-trifluoroethylene chlorofluoroethylene) terpolymer $(62.6 / 29.4 / 8 \mathrm{~mol}$. \%, 11-12 $\mu \mathrm{m}$ thick) is directly measured by infrared imaging at ambient conditions. The adiabatic temperature change is estimated to be $5.2 \mathrm{~K}$ for an applied electric field of $90 \mathrm{~V} / \mu \mathrm{m}$. The temperature change is independent of the operating frequency in the range of $0.03-0.3 \mathrm{~Hz}$ and is stable over a testing period of $30 \mathrm{~min}$. Application of this terpolymer is promising for micro-scale refrigeration. (C) 2014 AIP Publishing LLC.

[http://dx.doi.org/10.1063/1.4890676]
\end{abstract}

Micro-scale refrigeration systems are widely used for the cooling of integrated circuits, microelectromechanical sensors, and biomedical devices. ${ }^{1}$ Environment-friendly cooling technologies with a high efficiency are attractive due to growing energy demands and stringent environmental requirements. ${ }^{2}$ Although thermoelectric cooling is commonly applied and has been scaled to the micro-domain, ${ }^{3-6}$ the low efficiency and challenges in material fabrication suggest that alternatives are needed. ${ }^{6}$ While refrigeration based on the magnetocaloric effect $^{7}$ can be employed to achieve extremely low temperatures, miniaturization of devices is challenging while maintaining a high cooling performance due to the difficulty of realizing the large magnetic fields required. ${ }^{2}$

The electrocaloric (EC) effect is a phenomenon in which reversible, polarization-related temperature and entropy changes occur when an electric field is applied to certain materials. EC cooling, which operates on a refrigeration cycle analogous to magnetocaloric cooling, is an emerging technology. ${ }^{8}$ The highest reported adiabatic temperature change in a bulk EC material is $2.5 \mathrm{~K}$ at an electric field of $3 \mathrm{~V} / \mu \mathrm{m}$ and a temperature of $434 \mathrm{~K}$ for $\mathrm{Pb}_{0.99} \mathrm{Nb}_{0.02}\left(\mathrm{Zr}_{0.75} \mathrm{Sn}_{0.20} \mathrm{Ti}_{0.05}\right)_{0.98} \mathrm{O}_{3}{ }^{9}$. Recently, a large $\mathrm{EC}$ effect was discovered in thin films of $\mathrm{PbZr}_{0.95} \mathrm{Ti}_{0.05} \mathrm{O}_{3},{ }^{10} \mathrm{a}$ poly(vinylidene fluoride-trifluoroethylene) [P(VDF-TrFE)] copolymer, ${ }^{11}$ and a P(VDF-TrFE-chlorofluoroethylene) [P(VDFTrFE-CFE)] terpolymer. ${ }^{11}$ The P(VDF-TrFE-CFE) terpolymer demonstrates an adiabatic temperature change of $16 \mathrm{~K}$ at an electric field of $150 \mathrm{~V} / \mu \mathrm{m}$ near room temperature ${ }^{12}$ and is easily and economically fabricated, making it favorable for mass production. ${ }^{13}$ These findings point to the potential of applying EC cooling in micro-devices using polymer thin films.

Direct and indirect techniques can be applied to measure the EC effect. In the indirect measurement, a differential scanning calorimeter is used to measure the heat flow under a high electric field and isothermal conditions. ${ }^{14,15}$ This technique is best suited to bulk materials as the output heat flow signal for a thin film sample is very small. Jia and $\mathrm{Ju}^{16}$

\footnotetext{
${ }^{a} \mathrm{D}$. Guo and J. Gao contributed equally to this work.

b) scyao@cmu.edu.
}

reported an approach for characterizing the EC effect in a thin film sitting on an insulating substrate. In this approach, the temperature response of a resistance thermometer deposited on the bottom of the EC film is monitored as an electric field is turned on and off. In the reported measurements, the temperature change is less than $10 \%$ of that expected because the heat loss from the EC film to the substrate is large. Lu et al. ${ }^{17}$ employed a specially designed calorimeter to measure the EC effect in a thin film. In this approach, the heat generated in the EC film is compared with the heat generated from a standard reference resistor. More recently, an infrared camera was used to measure the temperature change in a $50 \mu \mathrm{m}$ thick polymer film under the direct application of an electric field. ${ }^{13}$

In this work, the infrared imaging technique, which enables high-frequency testing, is used to directly measure the transient temperature response of a P(VDF-TrFE-CFE) terpolymer thin film (11-12 $\mu \mathrm{m}$ thick) under varying electric fields near ambient conditions. The convection heat transfer between the thin film sample and the air is not negligible and must be included in the analysis. A significant percentage of the adiabatic temperature change (about 50\%) is still realized, however, which is much larger than that obtained in other techniques. ${ }^{14-16}$ The frequency dependence of the EC effect is investigated and the film stability is explored. X-ray diffraction (XRD) is employed to study the structural phase transition in the terpolymer when an electric field is applied.

Film samples from (i) Piezotech [France, P(VDF-TrFECFE): 62.6/29.4/8 mol. \%, $11 \mu \mathrm{m}$ thick] and Carnegie Mellon University (CMU, same composition as Piezotech samples, $12 \mu \mathrm{m}$ thick) were tested. The CMU films were fabricated by a solution-cast method following instructions from Piezotech. The polymer powder was first completely dissolved into methyl ethyl ketone and then the solution was cast on a silicon wafer and baked at a temperature of $50{ }^{\circ} \mathrm{C}$ for $12 \mathrm{~h}$ to evaporate the solvent. After drying, the film was immersed in water for $2 \mathrm{~h}$ and then peeled off the wafer. The film was then suspended and annealed at a temperature of $100^{\circ} \mathrm{C}$ for $7 \mathrm{~h}$ to remove residual solvent. ${ }^{12,13} 5 \mathrm{~mm}$-diameter gold circles of 
thickness $100 \mathrm{~nm}$ were sputtered on both sides of the film to act as the electrodes.

A large EC effect is rare in crystalline polymers. The required relaxor ferroelectric phase has only been observed in a few ferroelectric polymers due to their long-chains. ${ }^{18}$ The relaxor ferroelectric behavior is realized in the $\mathrm{P}(\mathrm{VDF}-\mathrm{TrFE}-$ $\mathrm{CFE}$ ) terpolymer at room temperature by incorporating the large CFE monomer to increase the interchain distance. ${ }^{11}$ Here, we performed in situ electric field-dependent $\theta-2 \theta$ XRD measurements for the P(VDF-TrFE-CFE) terpolymer at a temperature of $25^{\circ} \mathrm{C}$ and a reversible electric field-induced relaxor ferroelectric-ferroelectric phase transition is observed. ${ }^{19}$ We note that Yang et al. ${ }^{18}$ previously used two-dimensional wide-angle XRD to investigate crystal orientations in the $\mathrm{P}$ (VDF-TrFE-CFE) terpolymer film and a similar relaxor ferroelectric-ferroelectric phase transition was obtained by varying the temperature instead of the electric field.

The electric field-dependent temperature response of the terpolymer film is measured by infrared imaging. To obtain the temperature map accurately, a $2 \mathrm{~mm}$-diameter circular area at the center of the top electrode was coated with a $5 \mu \mathrm{m}$ layer of carbon ink to increase its emissivity to 0.7 . To measure the EC effect, the film was suspended $1 \mathrm{~mm}$ above a thermal stage in air. Voltage pulses were supplied by a function generator (Hewlett Packard 33120a) and amplified by a high-voltage amplifier (Trek 2210). The current and voltage were measured by a source meter (Keithley 2400) and a multimeter (Agilent 34401a) and were collected by LabVIEW. The temperature images were recorded by a QFI InfraScope II system in which the optical head includes a $256 \times 256$ element Indium-Antimonide infrared focal plane array detector that is cooled by liquid nitrogen. The sample rate is 14 frames per second. The temperature uncertainty of the infrared camera is $\pm 0.1{ }^{\circ} \mathrm{C}$ and the spatial resolution is $30 \mu \mathrm{m}$. The reported temperature is averaged over the ink-coated area.

Three Piezotech samples and three CMU samples were tested at a stage temperature of $25^{\circ} \mathrm{C}$ for maximum electric fields between 40 and $90 \mathrm{~V} / \mu \mathrm{m}$. For each test, three consecutive trapezoidal pulses with a period of $30 \mathrm{~s}$ were applied to check for repeatability. The ramp time of the voltage pulse was varied $(0.8,1.2$, and $1.6 \mathrm{~s})$ to allow for quantification of the heat transfer between the sample and the air environment. Typical results for the current and temperature responses are shown in Figures 1(a) and 1(b) and show a similar behavior. The temperature response induced by the electric field change happens very fast $(\sim$ milliseconds $){ }^{20}$ The sample temperature thus immediately rises when the electric field is turned on [1-2 in Figures 1(a) and 1(b)]. With the electric field still on, the temperature decreases exponentially back to the initial temperature due to the air cooling (2-3). When the electric field is turned off, the temperature quickly decreases below the initial temperature (3-4) and then rises exponentially back to the initial temperature (4-5). The current leakage for all samples is very small (10-30 nA when the voltage is applied for $15 \mathrm{~s})$. Joule heating in the film is thus negligible, consistent with the temperature result, in Figure 1(b) (i.e., $T_{1}=T_{3}$ ).

The measured cooling temperature induced by the EC effect, as shown in Figure 1(b), is plotted in Figure 2. (a)

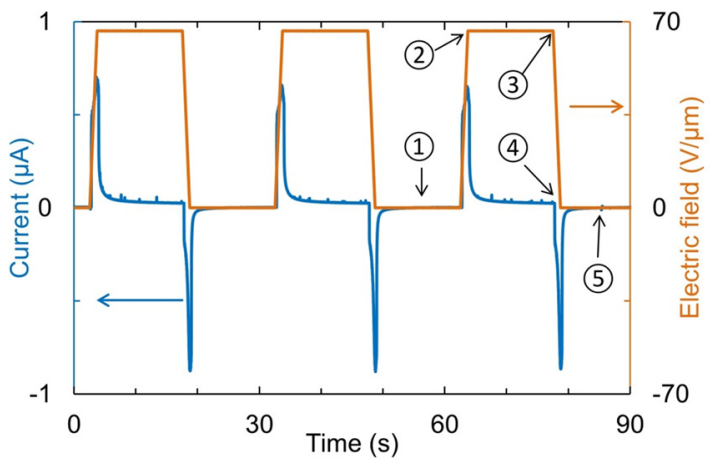

(b)
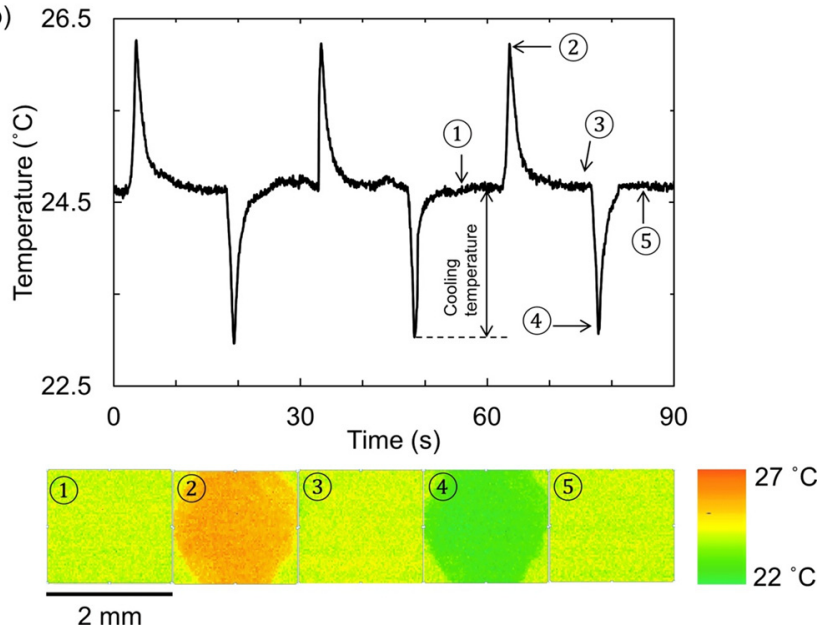

FIG. 1. (a) Example of a trapezoidal electric field applied to the EC sample and the measured current. The ramp time of the electric field is $1.2 \mathrm{~s}$ and its maximum amplitude is $67 \mathrm{~V} / \mu \mathrm{m}$. (b) Infrascope-measured temperature images at different times in a cycle and the temperature as a function of time. The temperature changes abruptly when the electric field is ramping up and down (2 and 4).

Consistent results are observed for the Piezotech and CMU samples. When the ramp time of the electric field is reduced, the cooling temperature increases as the heat loss to the air is less in a shorter ramp time. The adiabatic temperature changes of the terpolymer films were calculated by estimating the heat transfer between the sample and the air, ${ }^{19}$ and are plotted in Figure 3 along with literature results for the same material. The adiabatic temperature change over the

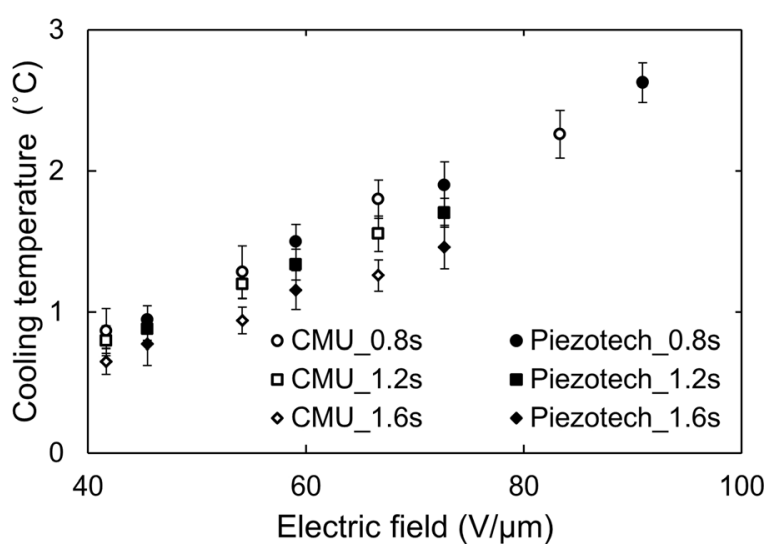

FIG. 2. Measured cooling temperature for CMU and Piezotech samples as a function of the electric field at different voltage ramp times. Same voltages are applied to CMU and Piezotech samples. Electric fields are different for them due to the thickness differences. The stage temperature is $25^{\circ} \mathrm{C}$ for all measurements. 


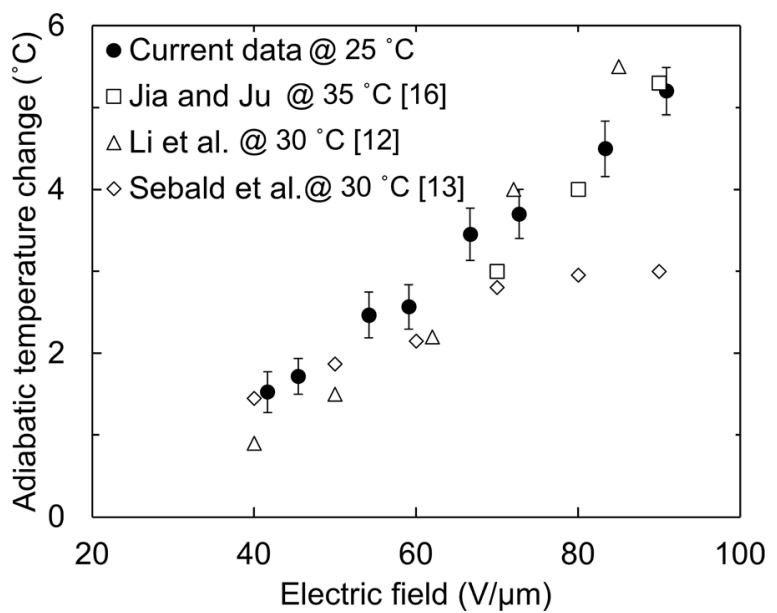

FIG. 3. Adiabatic temperature change vs. electric field and comparison to literature results.

full range of electric fields agrees well with the results of $\mathrm{Li}$ et al. ${ }^{12}$ and Jia and Ju. ${ }^{16}$ Deviations are observed with the data from Sebald et al., ${ }^{13}$ which saturate when the electric field exceeds $70 \mathrm{~V} / \mu \mathrm{m}$.

The temperature dependence of the EC effect in the $\mathrm{P}(\mathrm{VDF}-\mathrm{TrFE}-\mathrm{CFE})$ terpolymer is plotted in Figure 4 for stage temperatures between 20 and $30{ }^{\circ} \mathrm{C}$ and a voltage ramp time of $0.8 \mathrm{~s}$. The EC effect is increased by $10 \%-20 \%$ at a temperature of $30{ }^{\circ} \mathrm{C}$, consistent with the results reported in Ref. 12. The effect of the electric field frequency on the temperature change is shown in Figures 5(a) and 5(b) for frequencies between 0.03 and $0.3 \mathrm{~Hz}$. As shown in Figure 5(a), the minimum temperature (i.e., location 4 in Figure 2) increases with increasing frequency as the thermal time constant $(\sim 1 \mathrm{~s})$ becomes larger than the period of the operating frequencies. The inset in Figure 5(b) shows the temperaturetime history for a frequency of $0.3 \mathrm{~Hz}$. Note that the temperature does not return to the initial value during the air cooling/ heating stages and that an offset average temperature is realized. Thus, the maximum temperature decreases and the minimum temperature increases compared to lower-frequency operation. The measured cooling temperature $(\Delta T)$ induced by the EC effect when switching the electric field off is indicated in the inset figure, and this temperature change as a function of the frequency is plotted in Figure 5(b). The measured

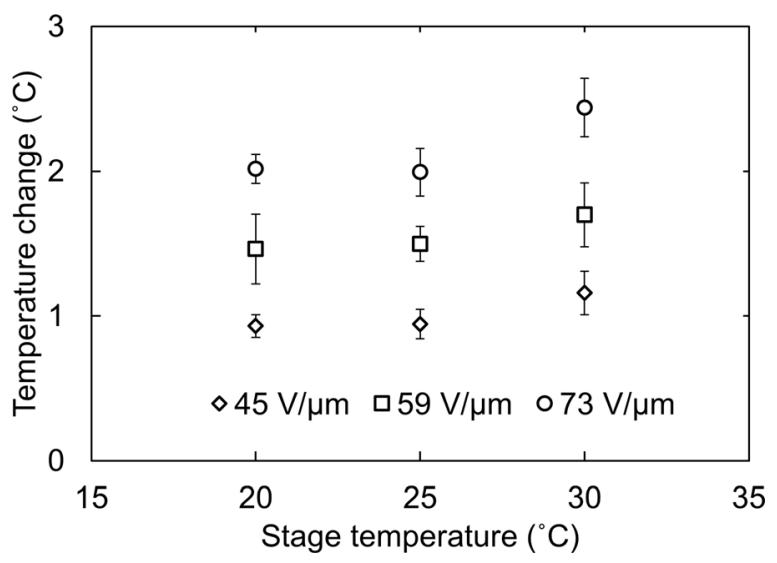

FIG. 4. Measured cooling temperature at different stage temperatures. The ramp time of the voltage is $0.8 \mathrm{~s}$. (a)

(b)
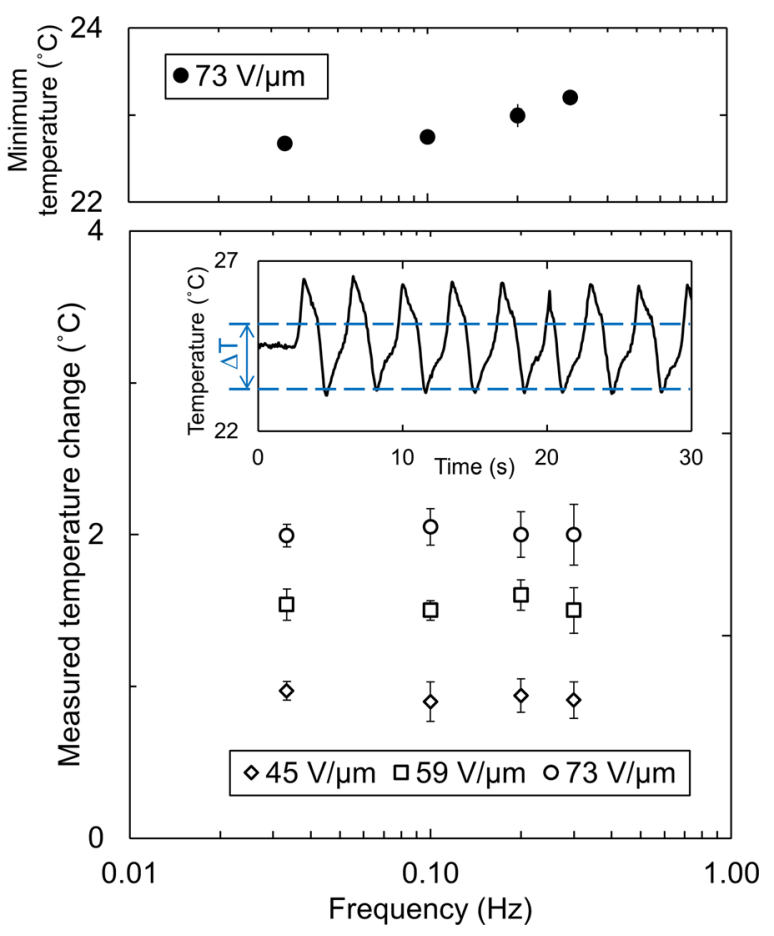

FIG. 5. (a) Measured minimum temperature as a function of the operating frequency for an electric field of $73 \mathrm{~V} / \mu \mathrm{m}$. (b) Measured temperature change when switching off the electric field vs. the operating frequency. A ramp time of $0.8 \mathrm{~s}$ is fixed at different frequencies for a fair comparison. The stage temperature is $25^{\circ} \mathrm{C}$. The inset is a plot of measured temperature when the operating frequency is $0.3 \mathrm{~Hz}$ and the electric field amplitude is $73 \mathrm{~V} / \mu \mathrm{m}$. $\Delta T$ is the temperature change when switching off the electric field.

temperature change does not change in the operating frequency range of $0.03-0.3 \mathrm{~Hz}$ (the infrascope limits us to this maximum frequency), demonstrating that the EC effect in the $\mathrm{P}(\mathrm{VDF}-\mathrm{TrFE}-\mathrm{CFE})$ terpolymer is frequency-independent. Our result is promising, in that a practical device will operate in a frequency range of $1-10 \mathrm{~Hz}$. For example, Gu et al. ${ }^{21}$ fabricated a solid-state chip-scale EC cooler using a P(VDF-TrFE) polymer and tested it at $1 \mathrm{~Hz}$.

The stability of the EC material was then assessed by conducting a 30 min test under continuous operation. The operating frequency was $0.2 \mathrm{~Hz}$ and the amplitude of the electric field was $54 \mathrm{~V} / \mu \mathrm{m}$ with a ramp time of $0.8 \mathrm{~s}$. The temperature history is plotted in Figure 6. The temperature

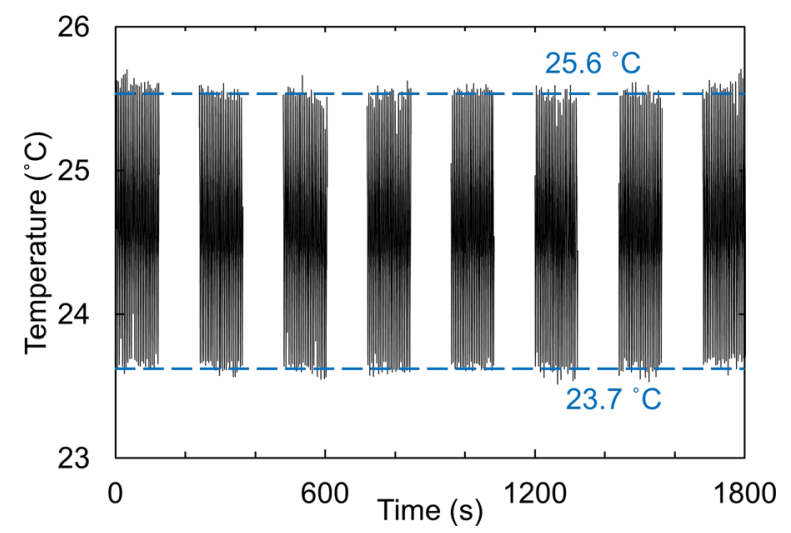

FIG. 6. Temperature history of a sample for $30 \mathrm{~min}$ at a stage temperature of $25^{\circ} \mathrm{C}$. The ramp time of the voltage is $0.8 \mathrm{~s}$. The frequency is $0.2 \mathrm{~Hz}$ and the electric field amplitude is $54 \mathrm{~V} / \mu \mathrm{m}$. Gaps in the date are due to limitations of the infrascope, the electric field was modulated at all times. 
data were not measured continuously due to the limitation of the infrascope. The time spacing between adjacent data collection periods is $2 \mathrm{~min}$. No failure was observed within $30 \mathrm{~min}$. The maximum and the minimum temperatures measured by the infrared camera are $25.6^{\circ} \mathrm{C}$ and $23.7^{\circ} \mathrm{C}$. The performance is stable and demonstrates the potential of applying the $\mathrm{P}(\mathrm{VDF}-\mathrm{TrFE}-\mathrm{CFE})$ polymer in a microcooler application.

In conclusion, the EC effect in thin films of a P(VDFTrFE-CFE) terpolymer was characterized by infrared imaging, which can be conducted conveniently and economically. The heat transfer from the sample to the ambient air was modeled and used to estimate the adiabatic temperature change at different electric field magnitudes. The electric field-induced structural phase transition in the terpolymer, which is critical in the EC effect, was identified using XRD. In an operating frequency range of $0.03-0.3 \mathrm{~Hz}$, the EC effect was observed to be independent of frequency. The stability of the material was validated by long time testing. All results suggest that this polymer is a promising material for micro-scale cooling application.

This work was supported by the Defense Advanced Research Projects Agency (DARPA) and the U.S. Army Aviation and Missile Research, Development, and Engineering Center (AMRDEC) under Grant No. W31P4Q10-1-0015. The views and conclusions contained in this document are those of the authors and should not be interpreted as representing the official policies, either expressed or implied, of DARPA, the U.S. Army, or the U.S. Government.
${ }^{1}$ C. Nadiya and R. C. Patel, Int. J. Software Hardware Res. Eng. 2, 38-41 (2014).

${ }^{2}$ M. Valant, Prog. Mater. Sci. 57, 980-1009 (2012).

${ }^{3}$ R. Venkatasubramanian, E. Siivola, T. Colpitts, and B. O'Quinn, Nature 413, 597-602 (2001).

${ }^{4}$ C. LaBounty, A. Shakouri, and J. E. Bowers, J. Appl. Phys. 89, 4059-4064 (2001).

${ }^{5}$ A. Majumdar, Science 303, 777-778 (2004).

${ }^{6}$ W. Kim, J. Zide, A. Gossard, D. Klenov, S. Stemmer, A. Shakouri, and A. Majumdar, Phys. Rev. Lett. 96, 045901 (2006).

${ }^{7}$ K. A. Gschneidner, Jr. and V. K. Pecharsky, Annu. Rev. Mater. Sci. 30, 387-429 (2000)

${ }^{8}$ D. Guo, J. Gao, Y. J. Yu, S. Santhanam, A. Slippey, G. K. Fedder, A. J. H. McGaughey, and S. C. Yao, Int. J. Heat Mass Transfer 72, 559-564 (2014).

${ }^{9}$ B. A. Tuttle and D. A. Payne, Ferroelectrics 37, 603-606 (1981).

${ }^{10}$ A. S. Mischenko, Q. Zhang, J. F. Scott, R. W. Whatmore, and N. D. Mathur, Science 311, 1270-1271 (2006).

${ }^{11}$ B. Neese, B. Chu, S. G. Lu, Y. Wang, E. Furman, and Q. M. Zhang, Science 321, 821-823 (2008).

${ }^{12}$ X. Li, X. S. Qian, S. G. Lu, J. Cheng, Z. Fang, and Q. M. Zhang, Appl. Phys. Lett. 99, 052907 (2011).

${ }^{13}$ G. Sebald, L. Seveyrat, J. F. Capsal, P. J. Cottinet, and D. Guyomar, Appl. Phys. Lett. 101, 022907 (2012).

${ }^{14}$ G. Sebald, L. Seveyrat, D. Guyomar, L. Lebrun, B. Guiffard, and S. Pruvost, J. Appl. Phys. 100, 124112 (2006).

${ }^{15}$ Y. Bai, G. Zheng, and S. Shi, Appl. Phys. Lett. 96, 192902 (2010).

${ }^{16}$ Y. Jia and Y. S. Ju, Appl. Phys. Lett. 103, 042903 (2013).

${ }^{17}$ S. G. Lu, B. Rožič, Q. M. Zhang, Z. Kutnjak, X. Li, E. Furman, L. J. Gorny, M. R. Lin, B. Malic, M. Kosec, R. Blinc, and R. Pirc, Appl. Phys. Lett. 97, 162904 (2010).

${ }^{18}$ L. Yang, X. Li, E. Allahyarov, P. L. Taylor, Q. M. Zhang, and L. Zhu, Polymer 54, 1709-1728 (2013).

${ }^{19}$ See supplementary material at http://dx.doi.org/10.1063/1.4890676 for XRD results of the terpolymer under different electric fields and the adiabatic temperature calculation process.

${ }^{20}$ T. Furukawa, IEEE Trans. Electr. Insul. 24, 375-394 (1989).

${ }^{21}$ H. Gu, X. Qian, X. Li, B. Craven, W. Zhu, A. Cheng, S. C. Yao, and Q. M. Zhang, Appl. Phys. Lett. 102, 122904 (2013). 